\title{
Towards a SiPM based fluorescence camera for JEM-EUSO
}

\section{Andreas Haungs*}

KIT - Karlsruhe Institute of Technology, IKP, 76021 Karlsruhe, Germany

E-mail: andreas.haungsekit.edu

\section{for the JEM-EUSO Collaboration}

The steady development of semiconductor devices in the last years has led to highly improved photon detectors (called SiPM) and with that their applicability for astroparticle physics experiments. Here, we discuss particularly the application for cosmic ray air-shower fluorescence telescopes in space (JEM-EUSO telescope). For this, improvements to the newest generation of SiPM are needed concerning the pixel dark current rate, optical crosstalk, and afterpulse rate. In addition, to be suitable for large-scale experiments in space like JEM-EUSO, temperature stability, operability in single photon counting, the fluorescence light sensitivity, as well as the capability of arrays of SiPM to cover large areas need to be rigorously tested. The JEM-EUSO collaboration started a comprehensive R\&D program ${ }^{\dagger}$ for the development of a focal surface based on SiPMs optimized for measurements of the fluorescence emission of extensive air showers from space.

The 34th International Cosmic Ray Conference,

30 July- 6 August, 2015

The Hague, The Netherlands

*Speaker.

${ }^{\dagger}$ Colleagues and institutions which have contributed to this paper are: M. Karus, T. Huber, F. Bisconti, H. Schieler, A. Weindl, A. Ebersoldt, KIT, Germany; P. Gorodetzky, APC, France; T. Jammer, J. Bayer, C. Tenzer, Tübingen, Germany; G. Osteria, Napoli, Italy; O. Catalano, Palermo, Italy; J. Estrada, S. Kerby, P. Rubinov, Fermilab, USA; E. Judd, Lawrence Berkeley National Lab, USA 


\section{Introduction}

The JEM-EUSO mission [1,2] aims to explore the origin and nature of the extreme energy cosmic rays (EECRs) through the observation of air-shower fluorescence light from space. The super-wide-field JEM-EUSO telescope will look down from the International Space Station onto the night sky to detect UV photons emitted during the passage in the atmosphere of the Extensive Air Showers (EAS) generated by the EECRs. Such a space detector offers a remarkable opportunity to observe a huge volume of atmosphere at once and will achieve unprecedented statistics within a few years of operation. Several test experiments are currently in operation: e. g. to observe the fluorescence background from the edge of the Atmosphere (EUSO-Balloon), and to demonstrate the capability of detecting air showers on the ground with an EUSO-type telescope (EUSO-TA).

\section{JEM-EUSO}

The instrument The entire JEM-EUSO instrument [3,4] consists of the telescope, the focal surface, a monitoring system for the atmospheric conditions and a calibration system. There will be support and calibration systems on the ground as well as at the ISS. The focal surface detector (Fig. 1) constitutes a $\mu$ s-fast and highly pixelized digital camera with a diameter of about $2.5 \mathrm{~m}$ and $\mathrm{a} \pm 30^{\circ}$ wide field of view. It works in the near-UV wavelength $(290-430 \mathrm{~nm})$ and enables single-photon counting. The baseline design of the focal surface will consist of 4932 64-channel multi-anode photomultiplier tubes from Hamamatsu. They will be organized in elementary cells (EC) of 4 MAPMTs each and in 137 photo detection modules (PDM) of 9 ECs each.

For fluorescence detection of cosmic rays it is essential to calibrate the detector pre-flight with utmost precision and to monitor the performance of the detector throughout the whole mission time. Calibration stands on-ground were built to measure precisely the performance of the photo sensors. They consist of photon-shielding, a uniform light source and readout electronics. The uniform light is available thanks to an integrating sphere and the light intensity is monitored in real-time by a NIST-calibrated photo diode attached to the sphere [5]. To investigate the suitability of alternative detector devices, further research is done with state-of-the-art silicon photomultipliers
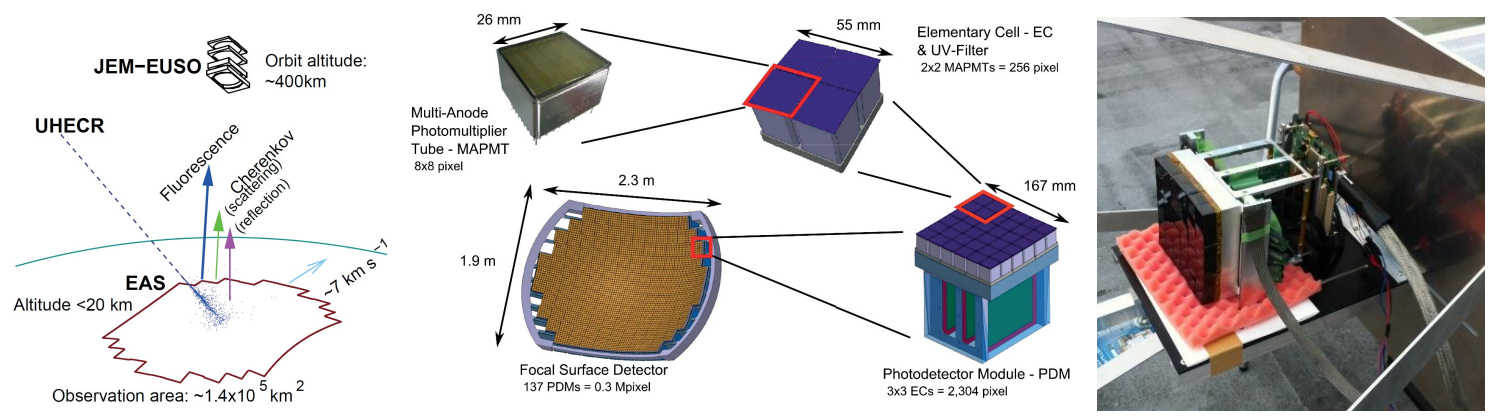

Figure 1: From left to right: i) Schematic view of the JEM-EUSO mission: EASs generate fluorescence light that is emitted isotropically as well as Cherenkov light emitted in forward direction. The latter is also scattered and reflected and can reach the aperture of the telescope [1]. ii) Schematic view of the focal surface and its components of the JEM-EUSO mission. iii) First fully assembled Photo Detector Module (PDM) for JEM-EUSO prototype experiments. 
(SiPMs). These will also be tested in the calibration stand and their performance can be compared with respect to conventional photomultiplier tubes.

Test experiments On the way to getting the full instrument for the launch to the ISS, several test or pathfinder experiments are needed and currently under development or in operation. A PDM (photon detection module with 2304 pixels, see Fig. 1) can be seen as an independent unit, and one of them will be used for most of the test experiments.

- EUSO-Balloon: EUSO-Balloon [6] is serving as a demonstrator for technologies and methods featured in the space instrument. This balloon-borne instrument points towards the nadir from a float altitude of about $40 \mathrm{~km}$. A first flight was performed on August, 25, 2014 at Timmins, Canada. A total of 5 hours of valuable data from the PDM and from the installed infrared camera, as well as Laser pulses sent by a helicopter accompanying the full flight are presently being analysed. In future more balloon flights are planned, including a NASA lead long duration flight with a super pressurized balloon (SPB-EUSO) [7].

- EUSO-TA: Important information on the capabilities of the full instrument is provided by EUSO-TA [8], which is a ground-based telescope formed by one PDM. EUSO-TA is located at the Telescope Array (TA) [9] site in Utah, USA. The instrument is triggered by the fluorescence detectors of TA, and will make use of the LIDAR and the Electron Light Source of TA.

- K-EUSO: Currently, part of the collaboration is considering a cooperation with the Russian project KLYPVE [10]. KLYPVE is a mirror based fluorescence telescope foreseen to be installed at the Russian segment of the ISS.

- Mini-EUSO: Another project is Mini-EUSO [11], a small prototype experiment (one PDM and a two-25cm-diameter Fresnel-lens system) foreseen to operate inside the ISS for an observation of the UV emission from the night-Earth through a UV-window. This instrument would map for the first time the Earth in UV, and could study atmospheric phenomena and bio-luminescence on Earth as well as meteors.

SiPM-EUSO In addition to the above mentioned test experiments the collaboration has also started an R\&D program to evaluate if SiPM can replace the heavy, HV-operated and expensive MAPMT's presently in use. The development plan includes several steps with the aim to complete the program on a time scale of 2-3 years.

- Development of an EC-like photo sensor based on SiPM's to be tested and further studied and improved in the laboratory.

- Integration of the SiPM-EC into the electronics and data stream of the JEM-EUSO test experiment devices and test measurements at these devices.

- Design and manufacture of an R\&D early prototype of a SiPM based PDM for the use in the test experiments.

- R\&D to apply the PDM in a space-based experiment

- Design study of a full SiPM focal surface detector as an advanced option for a future JEMEUSO like large-scale mission. 

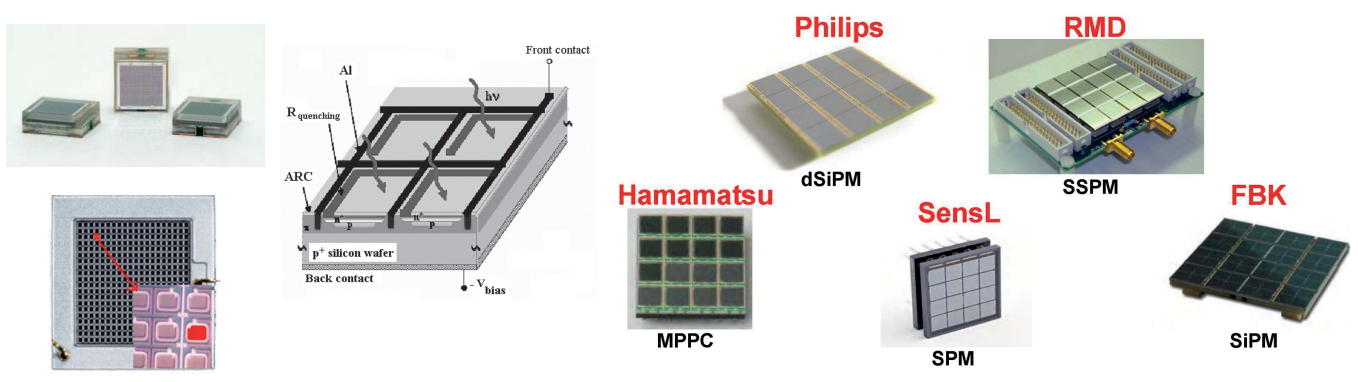

Figure 2: SiPM and arrays of SiPM of different sizes and companies (Taken from [13]).

\section{SiPM and JEM-EUSO}

A silicon photomultiplier is a relatively novel type of an optical semiconductor elemental device with photon counting ability. Due to the rapid commercial developments and following improvements and sensitivity, its application possibilities in astroparticle physics experiments increase from generation to generation. Every couple of months there are new version with improved characteristics on the market. Currently, considerable improvements happen much faster than for standard vacuum PMT. In general, SiPMs are compact, low cost, and low weight devices, have a high photon detection efficiency, a high gain, and a fast response. They also have the big advantage, compared to PMTs [12], of being able to operate at low voltages $(<100 \mathrm{~V})$, and without being affected by magnetic fields. In addition, they are more robust and therefore, a larger duty cycle for the measurements can be achieved.

A single SiPM pixel consists of multiple (several hundreds to several ten thousands) avalanche photodiodes (APDs), which are operated in Geiger mode. Commercially available from various companies are pixel sizes (of $1 \times 1 \mathrm{~mm}^{2}, 2 \times 2 \mathrm{~mm}^{2}$, or $3 \times 3 \mathrm{~mm}^{2}, \ldots$ ) or lately also arrays or strips of several pixels (see fig. 2). An G-APD is a high-speed and high-sensitivity photo-diode, whose photoelectric current is multiplied by applying a reverse bias voltage. Each G-APD pixel is usually operated in a binary mode, which outputs a fixed signal current, regardless of the detected number of photons. Hence, the output signal from a SiPM is the sum of charge of all the APD signals, which should be proportional to the number of the detected incident photons. However, dark current and crosstalk in reality hamper such a perfect view of SiPMs.

The most important parameter of photo sensors to be considered for JEM-EUSO is the photon detection efficiency (PDE), which depends on the transmittance of the surface, the filling factor of the sensitive area, the real quantum efficiency $(\mathrm{QE})$, and on the Geiger efficiency of the device.

Several kinds of SiPMs, possibly considerable as candidates for a JEM-EUSO focal surface detector, are currently being developed by Hamamatsu and other companies. They have a high sensitivity in the short-wavelength region. A promising candidate for a SiPM based focal surface is the new 64 Pixel TSV-MPPC array series from Hamamatsu (Through-Silicon Via Multi-Pixel Photo Counters) [14]. The feature of this new kind of SiPMs is that the anode and cathode are wired through the substrate.

With the objective of applying SiPM/MPPC to JEM-EUSO, we need (together with Hamamatsu) to further improve their photodetection efficiency at the device level, and quickly design the module and the prototype which have a more stable and safe operation in space. 


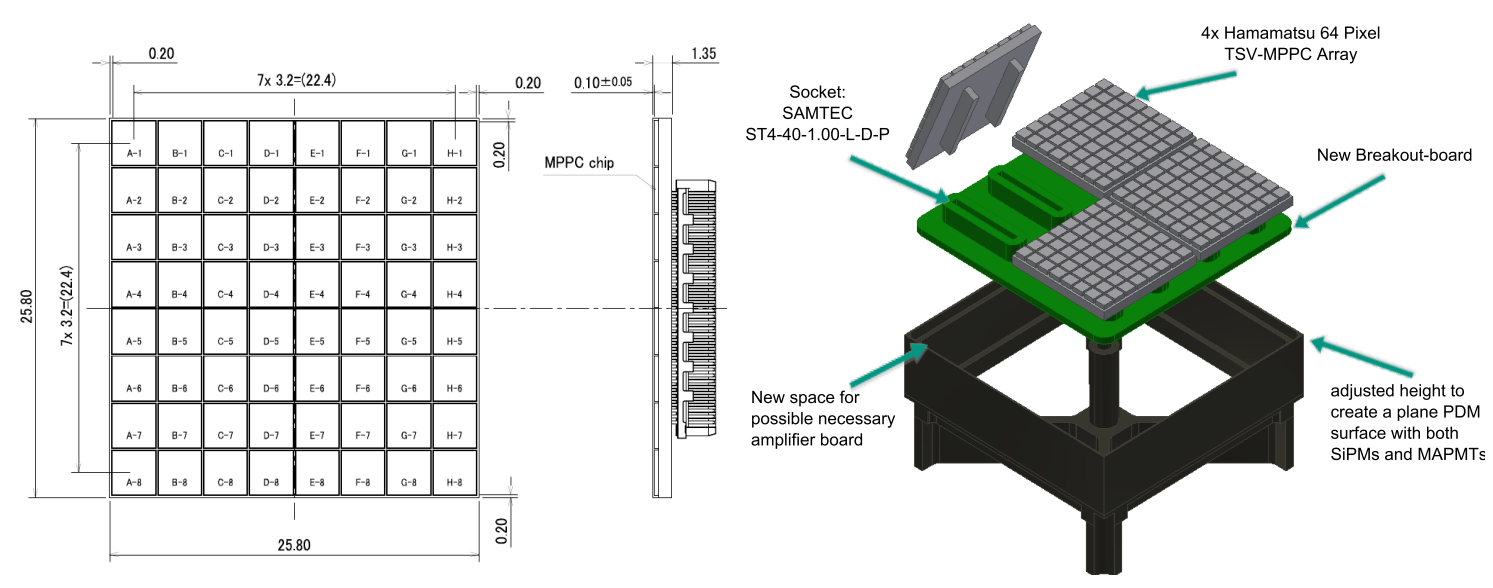

Figure 3: Left panel: Layout of the 64 pixel TSV-MPPC array S12642-0808PB-50. The geometrical dimension of four arrays fits with the former MAPMT-EC design [14]. Right: First prototype of a SiPM based EC. Due the more compact structure of TSV-MPPCs there is new space for further electronics like temperature measurements or an amplifier board.

\section{SiPM for JEM-EUSO: a comprehensive R\&D study}

To reach the goal of providing an EC based on SiPM within the next 1 to 2 years the following main issues have to considered: (i) a large sensitive area with a high filling factor needs to be provided to avoid dead space and the usage of light cones; (ii) presently available SiPMs need an improvement in their sensitivity to fluorescence light (UV-range around $300 \mathrm{~nm}$ ); (iii) a fast readout has to be developed (e.g. specific ASIC, digital SiPM, monolithic SiPM/ASIC readout); and (iv) the characteristics of the new generation SiPMs have to be determined and their calibration performed (single photon efficiency). In more details, we are considering the following work packages within these studies.

Characteristics of different SiPMs As mentioned above there is a worldwide effort on developments of improved SiPMs by various companies [15]. Available new devices need to be tested and their characteristics need to be validated by detailed laboratory measurements. Many institutes that are members of the JEM-EUSO Collaboration have the equipment to perform such tests. Most important is the characterization of SiPM with respect to the following parameters: (i) Dark current. In the advanced design of JEM-EUSO, the environmental temperature of JEM-EUSO can be decreased to $-15^{\circ} \mathrm{C} \pm 2-3^{\circ} \mathrm{C}$, therefore the noise rate caused by the dark current of the SiPM should be low enough in this temperature range. (ii) Stability of gain. SiPM normally have around $3 \% /{ }^{\circ} \mathrm{C}$ of temperature dependence of the gain, however, we need to limit it till around $-0.1 \% /{ }^{\circ} \mathrm{C}$, which is possible by using an adequate thermistor as temperature compensation circuit. We will design the detection module so that the temperature of SiPM inside is uniform and stable. (iii) Crosstalk. Improvements are given, but this parameter has to be determined individually for the new devices.

Going for single photon calibration Important for the low light measurements in space are the good single photon detection capability and the stability of the PDE. The intrinsic dark count rate of SiPMs is in the range of a few MHz at room temperature, and in addition, there is a lot of optical 

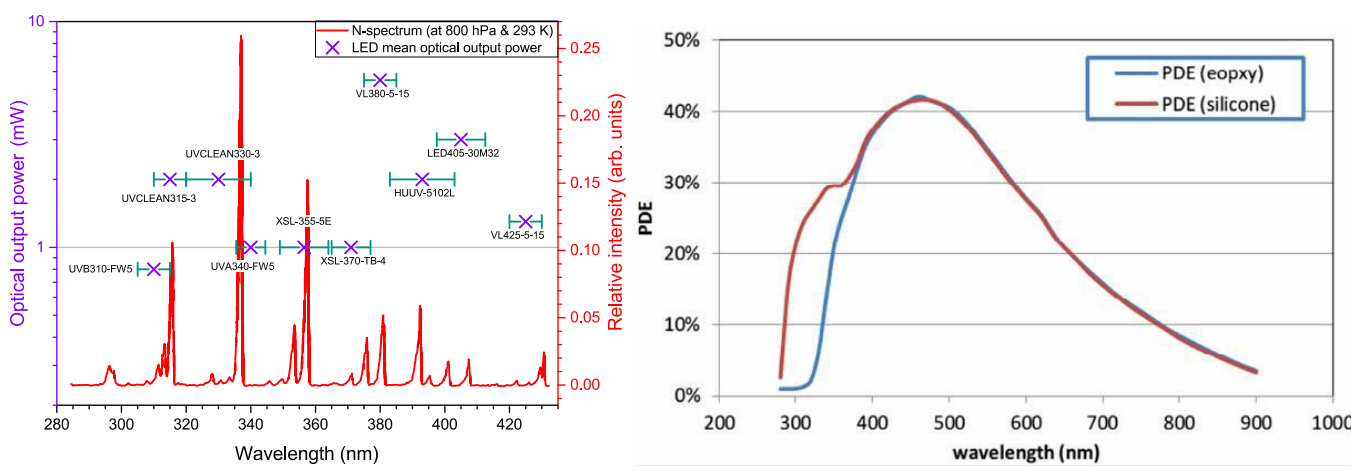

Figure 4: Left panel: Fluorescence spectrum with an overlay of UV-LED optical outputs (N-spectrum adopted from [16]). Right panel: from the Hamamatsu data sheet of the new TSV arrays with different coatings.

crosstalk and thermal noise polluting the spectrum. Hence, a determination of the efficiency via the single photoelectron spectrum alone does not work [17]. For SiPMs the detection of photons follows Poisson statistics. From that the possibility to detect a photon can be expressed via the possibility to not detect any photon (pedestal) and the possibility to detect noise. A dark spectrum and a light spectrum (up to a level of 410 photoelectrons) have to be measured. Integrating the pedestals of both spectra gives the number of events in the pedestals. The number of traces (dark spectrum) and respectively the trigger counter (light spectrum) provide the number of all events in each spectrum $\left(N_{\text {tot }}^{\text {dark }}\right.$ and $\left.N_{\text {tot }}\right)$ from which the number of detected photons can be calculated. The efficiency of the SiPM is given by the ratio of the detected photons $n_{\mathrm{pe}}$ versus the number of single photons sent on the device. For the purpose of a more versatile calibration set-up a new LED-array was designed, to allow PDE measurements with different wavelengths. The resulting light source is a spectral light source covering the range of $310-425 \mathrm{~nm}$ with several LEDs (Fig. 4, left panel). In such a way the main peaks of the nitrogen fluorescence spectrum are covered and the PDE can be measured for different wavelengths.

Going for larger arrays of SiPM The brand new TSV-arrays of Hamamatsu have now reached a geometrical filling factor of $84 \%$ which is already better than the baseline MAPMT used for the JEM-EUSO focal surface detector (see Fig. 3). This TSV technique allows us to reduce the dead space between two pixels from $3 \mathrm{~mm}$ to $0.2 \mathrm{~mm}$ so that there is no more need to mount Winston cones.

Going for UV sensitivity The fluorescence spectrum has the main peaks in the range $310-$ $430 \mathrm{~nm}$. MAPMTs and SiPMs have different wavelength sensitivity ranges. Indeed the sensitive range for MAPMTs is $280-650 \mathrm{~nm}$ while for SiPMs it is $320-900 \mathrm{~nm}$. To test what the detector's response in front of an EAS would be, some simulations have been done considering SiPMs instead of MAPMTs. They were made using the analysis and simulation framework Offline [18], created for the Pierre Auger Observatory and then adapted to the needs of the JEM-EUSO experiment [19]. In the case of SiPMs the photons from the first peak of the fluorescence spectrum are not detected. MAPMTs seem to be slightly better than SiPMs [5]. However, if the sensitivity range of SiPMs is improved, they would be probably better than MAPMTs. In Fig. 4, right panel the PDE of the new 
version of Hamamatsu TSV MPPCs, which is realized with a different coating, is displayed. These new SiPM arrays will be available at the end of 2015 .

Going for temperature control The temperature dependence of the SiPM gain is one of the most important topics to consider in the case of SiPM application to JEM-EUSO. The temperature dependence of the gain is $\sim-3 \%$ per ${ }^{\circ} \mathrm{C}$ at a certain bias voltage. A bias voltage compensation circuit using a thermistor which enables us to reduce the temperature coefficient down to around $0.3 \%$ per ${ }^{\circ} \mathrm{C}$, equivalent to a PMT is, however, easily possible. On the other hand, the behavior of the new TSV-MPPCs at low temperatures is not well known. Several efforts are presently being made to investigate the performance of TSV-MPPCs with temperature change. We start by measuring the dark current and the dark noise of TSV-MPPCs to compare the results with the characteristics of the old ceramic packed MPPCs. Operation at low temperatures with a good PDE is generally possible for SiPMs [20].

Going for dynamic range Beside the shower measurements, where only a few photons per pixel need to be measured, a large part of the JEM-EUSO program is to measure very intense light. This concerns e.g. lightning, Transient Luminous Events TLEs, meteors, space debris, etc. The light intensity can then be as high as a million times the single photoelectron level requiring a high dynamic range. To measure the dynamic range of a SiPM, we must be able to control the light in terms of number of photons per surface per second, which is possible with our calibration stands. In addition, we will measure accurately $(1 \mathrm{mV})$ the voltage and the overvoltage given to the SiPM. The current must be read with an accuracy of $1 \mu \mathrm{A}$ and must be limited to $<100 \mu \mathrm{A}$.

Going for fast readout We need to design carefully the part which transmits the pulse to the readout circuit considering sufficiently the following points: impedance matching between SiPM/ MPPC, degradation of the time character of the pulse due to floating capacitance, increase of electric noise accompanied with the implementation and electric interference among channels. Most probably these requirements need the development of a dedicated ASIC, preferably already integrated with the SiPM sensor (monolithic design). Companies as well as research groups are working currently in this direction.

Going for simulations In parallel to the hardware activities the performance of JEM-EUSO and its test experiments under the option of SiPM based focal surface detectors will be simulated in details as it was/is done for the JEM-EUSO baseline design [1].

Going for integration as EC A first CAD design of a prototype SiPM based EC (see Fig. 3) was made. The candidate sensor 64-pixel TSV MPPC array from Hamamatsu fits mechanically well into the former design of the MAPMT based EC. A 3-D model of such an EC was printed for further mechanical tests. The size of one 64-pixel TSV MPPC array is $25.8 \mathrm{~mm}$ to $25.8 \mathrm{~mm}$ compared to the MAPMTs with $26.2 \mathrm{~mm}$ to $26.2 \mathrm{~mm}$. Early results indicate that it will be possible to create a PDM with MAPMT-ECs and TSV-MPPC-ECs side by side in the foreseeable future.

Going for integration in PDM A concept to integrate SiPM ECs in the standard DAQ and readout system of an existing PDM will be part of this working package. 
Going for vibration tests The new arrays of TSV-MPPCs are mechanically differently fabricated compared to the older generations. Thorough tests need to be performed to see if they fulfil all requirements for a space qualification.

Going for radiation tests Radiation might cause the increase of the dark current of SiPMs, and a degradation of the resolution, because the collision of protons or neutrons produces defects in the lattice structure of the silicon crystals. There is a small influence due to electrons and $\gamma$-rays. Radiation tolerance tests have already been done, however, new arrays need to be tested.

\section{Conclusions}

The JEM-EUSO collaboration is performing a comprehensive study on the newest generation of SiPMs, where the goal is to set-up an Elementary Cell like sub-detector within 1-2 years. This will be used to compare the performance of both detector types (MAPMT and SiPM) in the laboratories as well as in EUSO test experiments like Mini-EUSO, EUSO-TA and EUSO-Balloon. Because of the reduced size and lower mass of SiPMs compared to MAPMTs, the SiPM solution can also be considered as an advanced option for the focal surface detector of the future large-scale mission in preparation.

Acknowledgment: This work was partially supported by the Italian Ministry of Foreign Affairs, General Direction for the Cultural Promotion and Cooperation, by the 'Helmholtz Alliance for Astroparticle Physics HAP' funded by the Initiative and Networking Fund of the Helmholtz Association, Germany, and by NASA award 11-APRA-0058 in the USA.

\section{References}

[1] J.H. Adams Jr. et al. - JEM-EUSO Collaboration, Astroparticle Physics 44 (2013) 76

[2] A. Haungs et al. - JEM-EUSO Collaboration, EPJ Web Conf. 52 (2013) 06005

[3] M. Casolino et al. - JEM-EUSO Collaboration, Exp. Atron. DOI 10.1007/s10686-014-9418-x (2014)

[4] F. Kajino et al., Nucl. Inst. Meth. A $\mathbf{6 2 3}$ (2010) 422

[5] M. Karus et al. - JEM-EUSO Collaboration, Proc. 34th ICRC (2015) PoS(ICRC2015)612

[6] P. von Ballmoos et al. - JEM-EUSO Collaboration, Proc. 34th ICRC (2015) PoS(ICRC2015)322

[7] L. Wiencke et al. - JEM-EUSO Collaboration, Proc. 34th ICRC (2015) PoS(ICRC2015)631

[8] M. Casolino et al. - JEM-EUSO Collaboration, Proc. 34th ICRC (2015) PoS(ICRC2015)636

[9] T. Abu-Zayyad et al. - Telescope Array Collaboration, Astropart. Phys. 39 (2013) 109

[10] M. Panasyuk et al. - JEM-EUSO Collaboration, Proc. 34th ICRC (2015) PoS(ICRC2015)669

[11] M. Ricci et al. - JEM-EUSO Collaboration, Proc. 34th ICRC (2015) PoS(ICRC2015)599

[12] V. D. Kovaltchouk et al., NIMA $\mathbf{5 3 8}$ (2005) 408

[13] R. Mirzoyan, contribution to a photo sensor workshop (2015), private communication

[14] Hamamatsu, TSV MPPC Array S12642-0808PB-50, Datasheet, (2014).

[15] APPEC, see http://www.appec.org/, workshop on photo sensors, München, April (2015)

[16] M. Ave et al. - AIRFLY Collaboration, NIM A 597 (2008) 41.

[17] P. Eckert et al., NIM A 620 (2010) 217

[18] S. Argiro et al. - Pierre Auger Collaboration NIM A 580 (2007) 1485

[19] T. Paul et al. - JEM-EUSO Collaboration, Proc. 34th ICRC (2015) PoS(ICRC2015)578

[20] P. K. Lightfoot et al., JINST 3 (2008) P10001 